\title{
Identification of key genes, pathways and potential therapeutic agents for liver fibrosis using an integrated bioinformatics analysis
}

\author{
Zhu Zhan ${ }^{1,2}$, Yuhe Chen ${ }^{1,2}$, Yuanqin Duan ${ }^{1,2}$, Lin Li ${ }^{3}$, Kenley Mew ${ }^{4}$, Peng Hu ${ }^{1,2}$, Hong Ren ${ }^{1,2}$, Mingli \\ Peng Corresp. 1,2 \\ ${ }^{1}$ Key Laboratory of Molecular Biology for Infectious Diseases (Ministry of Education), Chongqing Medical University, Chongqing, China \\ 3 Department of hepatic disease, Chongqing Traditional Chinese Medicine Hospital, Chongqing, China \\ 4 Department of foreign language, Chongqing Medical University, Chongqing, China \\ Corresponding Author: Mingli Peng \\ Email address: Peng_mingli@hospital.cqmu.edu.cn
}

Background. Liver fibrosis is often a consequence of chronic liver injury, and has the potential to progress to cirrhosis and liver cancer. Despite being an important human disease, there are currently no approved anti-fibrotic drugs. In this study, we aim to identify the key genes and pathways governing the pathophysiological processes of liver fibrosis, and to screen therapeutic anti-fibrotic agents. Methods. Expression profiles were downloaded from the Gene Expression Omnibus (GEO), and differentially expressed genes (DEGs) were identified by R packages (Affy and limma). Gene functional enrichments of each dataset were performed on the DAVID database. Protein-protein interaction (PPI) network was constructed by STRING database and visualized in Cytoscape software. The hub genes were explored by CytoHubba plugin app and validated in another GEO dataset and in a liver fibrosis cell model by quantitative real-time PCR assay. The Connectivity Map L1000 platform was used to identify potential anti-fibrotic agents. Results. We integrated 3 fibrosis datasets of different disease etiologies, incorporating a total of 70 severe (F3-F4) and 116 mild (F0-F1) fibrotic tissue samples. Gene functional enrichment analyses revealed that cell cycle was a pathway uniquely enriched in a dataset from those patients infected by hepatitis B virus (HBV), while the immune-inflammatory response was enriched in both the HBV and hepatitis $\mathrm{C}$ virus (HCV) datasets, but not in the nonalcoholic fatty liver disease (NAFLD) dataset. There was overlap between these three datasets; 185 total shared DEGs that were enriched for pathways associated with extracellular matrix constitution, platelet-derived growth-factor binding, protein digestion and absorption, focal adhesion, and PI3K-Akt signaling. In the PPI network, 25 hub genes were extracted and deemed to be essential genes for fibrogenesis, and the expression trends were consistent with GSE14323 (an additional dataset) and liver fibrosis cell model, confirming the 
relevance of our findings. Among the 10 best matching anti-fibrotic agents, Zosuquidar and its corresponding gene target ABCB1 might be a novel anti-fibrotic agent or therapeutic target, but further work will be needed to verify its utility. Conclusions. Through this bioinformatics analysis, we identified that cell cycle is a pathway uniquely enriched in HBV related dataset and immune-inflammatory response is clearly enriched in the virus-related datasets. Zosuquidar and ABCB1 might be a novel anti-fibrotic agent or target. 


\section{Identification of key genes, pathways and potential}

2 therapeutic agents for liver fibrosis using an

3 integrated bioinformatics analysis

4

5 Zhu Zhan ${ }^{1,2}$, Yuhe Chen ${ }^{1,2}$, Yuanqin Duan ${ }^{1,2}$, Lin $\mathrm{Li}^{3}$, Kenley Mew ${ }^{4}$, Peng $\mathrm{Hu}^{1,2}$, Hong Ren ${ }^{1,2}$,

6 Mingli Peng ${ }^{1,2}$

7

$8 \quad{ }^{1}$ Key Laboratory of Molecular Biology for Infectious Diseases (Ministry of Education),

9 Chongqing Medical University, Chongqing, China

$10{ }^{2}$ Department of Infectious Diseases, The Second Affiliated Hospital of Chongqing Medical

11 University, Chongqing, China

$12{ }^{3}$ Department of hepatic disease, Chongqing Traditional Chinese Medicine Hospital, Chongqing,

13 China

$14{ }^{4}$ Department of foreign language, Chongqing Medical University, Chongqing, China

17 Corresponding Author:

18 Mingli Peng 1

19 NO. 1, Yixueyuan Road, Yuzhong District, Chongqing, China.

20 Email address: Peng_Mingli@hospital.cqmu.edu.cn 


\section{Abstract}

24 Background. Liver fibrosis is often a consequence of chronic liver injury, and has the potential to progress to cirrhosis and liver cancer. Despite being an important human disease, there are currently no approved anti-fibrotic drugs. In this study, we aim to identify the key genes and pathways governing the pathophysiological processes of liver fibrosis, and to screen therapeutic anti-fibrotic agents.

Methods. Expression profiles were downloaded from the Gene Expression Omnibus (GEO), and differentially expressed genes (DEGs) were identified by R packages (Affy and limma). Gene functional enrichments of each dataset were performed on the DAVID database. Protein-protein interaction (PPI) network was constructed by STRING database and visualized in Cytoscape software. The hub genes were explored by CytoHubba plugin app and validated in another GEO dataset and in a liver fibrosis cell model by quantitative real-time PCR assay. The Connectivity Map L1000 platform was used to identify potential anti-fibrotic agents.

Results. We integrated 3 fibrosis datasets of different disease etiologies, incorporating a total of 70 severe (F3-F4) and 116 mild (F0-F1) fibrotic tissue samples. Gene functional enrichment analyses revealed that cell cycle was a pathway uniquely enriched in a dataset from those patients infected by hepatitis B virus (HBV), while the immune-inflammatory response was enriched in both the HBV and hepatitis $\mathrm{C}$ virus (HCV) datasets, but not in the nonalcoholic fatty liver disease (NAFLD) dataset. There was overlap between these three datasets; 185 total shared DEGs that were enriched for pathways associated with extracellular matrix constitution, plateletderived growth-factor binding, protein digestion and absorption, focal adhesion, and PI3K-Akt signaling. In the PPI network, 25 hub genes were extracted and deemed to be essential genes for fibrogenesis, and the expression trends were consistent with GSE14323 (an additional dataset) and liver fibrosis cell model, confirming the relevance of our findings. Among the 10 best matching anti-fibrotic agents, Zosuquidar and its corresponding gene target ABCB1 might be a novel anti-fibrotic agent or therapeutic target, but further work will be needed to verify its utility. Conclusions. Through this bioinformatics analysis, we identified that cell cycle is a pathway uniquely enriched in HBV related dataset and immune-inflammatory response is clearly enriched in the virus-related datasets. Zosuquidar and ABCB1 might be a novel anti-fibrotic agent or target. 


\section{Introduction}

64 Hepatic fibrosis is characterized by the pathological accumulation of extracellular matrix (ECM)

65

66

67

68

69

70

71

72

73

74

75

76

77

78

79

80

81

82

83

84

85

86

87

88

89

90

91

92

93

94

95

96

97 following chronic liver injury arising from various sources including toxic damage, viral infections, autoimmune conditions, and metabolic or genetic diseases. Patients with advanced liver fibrosis generally have a poor prognosis as they often develop decompensated cirrhosis and hepatocellular carcinoma (Tsochatzis et al. 2014).

For the management of patients with hepatic fibrosis in clinical practice, several wellvalidated clinical practice guidelines and recommendations have been established, including antiviral therapy for patients with chronic hepatitis B or C (Liver 2017a; Liver 2017b), a cessation of alcohol consumption in patients with alcoholic liver disease (Liver 2012), and lifestyle modifications in patients with nonalcoholic fatty liver disease (Djordjevic et al. 2018). However, eliminating the cause of fibrosis is not generally sufficient to halt progression from liver fibrosis to cirrhosis (Feng et al. 2018). Unfortunately, there are no approved anti-fibrotic drugs currently available (Bottcher \& Pinzani 2017). A better understanding of the molecular mechanisms controlling the fibrotic response is thus needed to facilitate the development of new drugs, and to thereby improve patient outcomes.

High-throughput sequencing technology offers an ideal means of profiling large gene expression datasets in order to gain a comprehensive understanding of the mechanisms underlying fibrosis. For example, Chan et al. found that in cirrhotic liver tissues there is a unique gene expression pattern related to inflammation, the immune response, and cell growth, and with a potential relationship with cancer as well (Chan et al. 2016). Using a bioinformatics analysis, many hub genes which are essential to fibrogenesis have been identified. For example, ITGBL1 was identified in an HBV-related fibrosis dataset (Wang et al. 2017). LUM, THBS2, FBN1, and EFEMP1 were all identified in a NAFLD-related fibrosis dataset (Lou et al. 2017). TAF1, HNF4A and CALM2 were identified in an HCV-related fibrosis dataset (Ji et al. 2018). COL6A1, COL6A2, COL6A3, PIK3R3, COL1A1, and CCND2, were identified in NAFLD and HCVrelated datasets (Chen et al. 2017). However, it remains unclear as to whether these pathways and hub genes are unique to individual disease etiologies or are shared between them.

In order to clarify this uncertainty, we integrated three datasets, each pertaining to fibrosis of a different etiology. Using bioinformatics analyses, we thereby sought to identify key genes and pathways of interest, and to screen for therapeutic agents and novel targets with the potential to treat liver fibrosis. 


\section{Materials \& Methods}

\section{Microarray data.}

100 Four gene expression datasets were downloaded from the Gene Expression Omnibus (GEO)

101 database; three were analyzed to identify DEGs, while one was used for validation. Table 1

102 summarizes the pertinent information for the selected GEO datasets used in this study. GSE6764

103 (Wurmbach et al. 2007), GSE49541(Moylan et al. 2014), and GSE84044 (Wang et al. 2017)

104 represent datasets from patients with liver fibrosis arising from hepatitis $\mathrm{C}$ virus (HCV),

105 nonalcoholic fatty liver disease (NAFLD), and hepatitis B virus (HBV), respectively. All three of

106 these gene expression profiles were based on the GPL570 platform. GSE49541 and GSE84044

107 were derived from two liver fibrosis studies in which tissues with severe fibrosis (F3-F4) and

108 mild fibrosis (F0-F1) were selected. GSE6764 and GSE14323 (Mas et al. 2009) (used for

109 validation) were derived from two liver cancer studies, in which cirrhotic (F4) and normal tissues

110 (F0) were selected.

111

Identification of differentially expressed genes.

Background expression value correction and data normalization were conducted for the raw data in each dataset using an R package (Affy, version 1.52.0). Probes in each data file were then annotated based on the appropriate platform annotation files. Probes without matching gene symbols were removed. In instances where different probes mapped to the same gene, the mean value of all probes mapping to that gene was taken as the final expression value for that gene. Then, the Linear Models for Microarray Analysis R package (limma; version 3.30.11) was applied for differential expression analysis. Those genes with an adjusted $\mathrm{P}$-value $<0.05$ and absolute value of fold-change $(\mathrm{FC})>1.5$ were deemed to be the DEGs. DEGs overlapping between datasets were obtained using an online Venn analysis tool (http://bioinformatics.psb.ugent.be/webtools/Venn/).

123

\section{Gene Ontology and pathway enrichment analyses.}

Gene Ontology (GO) is a commonly used bioinformatics tool that provides comprehensive information on gene function of individual genomic products based on defined features. This analysis consists of three facets: molecular functions (MF), biological processes (BP) and cellular components (CC). The Kyoto Encyclopedia of Genes and Genomes (KEGG) is a database resource for understanding high-level biological functions and utilities. These analyses and annotations are based on the DAVID database (https://david.ncifcrf.gov/), which provides a comprehensive set of functional annotation tools for investigators to explore and understand the biological meaning underlying particular gene lists. In this study, both GO and KEGG analyses of DEGs were performed with a criterion false discovery rate $(\mathrm{FDR})<0.05$.

\section{Protein-protein interaction (PPI) network construction and hub gene analysis.}


138 a database of known and predicted protein-protein interactions, and the results with a minimum

139

140

141

142

143

144

145

146

147

148

149

150

151

152

153

154

155

156

157

158

159

160

161

162

163

164

165

166

167

168

169

170

171

172

173

174

175

176

177

interaction score of 0.4 were visualized in Cytoscape. Furthermore, CytoHubba, a Cytoscape plugin app, providing a user-friendly interface to explore important nodes in biological networks, was utilized with the maximal clique centrality (MCC) method to explore the PPI network for hub genes.

\section{DEGs Validation.}

Another dataset GSE14323 was used to confirm the validity and disease relevance of identified DEGs. A heat map of the expression of 25 hub genes was developed using the HemI1.0.3.3 software. Statistical difference analysis between the liver cirrhosis group (LC) and normal control group (NC) was performed via student's t-test using SPSS V20.0. $P<0.05$ was considered statistically significant. As activation of hepatic stellate cells (HSCs) is considered as a central driver of liver fibrosis, we used a human HSC cell line - LX2 treated with TGF- $\beta 1$ to represent this activation stage. An expression of 25 hub genes was performed by quantitative real-time PCR assay compared with normal control.

\section{Cell culture and treatment.}

The LX2 cell line was purchased from Procell Life Science \& Technology (Wuhan, China), cultured with Dulbecco Modified Eagle Medium (DMEM)-high glucose supplemented with 10\% fetal bovine serum (FBS) and antibiotics (100 U/mL penicillin-G and $100 \mu \mathrm{g} / \mathrm{mL}$ streptomycin), and incubated at $37^{\circ} \mathrm{C}$ in $5 \% \mathrm{CO} 2$ and $95 \%$ humidified air. The LX2 cells were seeded in a 10$\mathrm{cm}$ culture dish at a density of $1 * 10^{6}$ for 6 hours. After attachment, the LX2 cells were treated with TGF- $\beta 1$ (R\&D systems, Catalog \#240-B/CF) at $10 \mathrm{ng} / \mathrm{ml}$ concentration or left untreated as normal control for 24 hours. Then RNA and proteins were isolated for further use.

\section{Western blot assay.}

LX2 cells total protein were extracted with ice-cold RIPA lysis buffer. Protein concentration was determined using the BCA Protein Assay Kit (Thermo Fisher Scientific, USA). Quantified proteins were separated on SDS-PAGE and transferred onto PVDF membranes (Millipore Corporation, USA). After blocking, membranes were incubated with anti- $\alpha$ SMA (1:20000, ab124964, Abcam, UK) at $4{ }^{\circ} \mathrm{C}$ overnight. Then, membranes were washed with TBST and incubated with secondary antibodies for 2 hours at room temperature. The anti-GAPDH $(1: 1000$, CST) was set as internal control. Protein bands were visualized by using ECL equipment (Pierce Chemical, USA).

\section{Quantitative real-time PCR assay.}

RNA was extracted from cell line LX2 by Trizol reagent (Takara, Japan) by following the manufacturer's instructions. The cDNAs were synthesized with a commercial kit (Takara, Japan). Gene expressions were measured by real-time PCR with CFX Connect ${ }^{\mathrm{TM}}$ Real-Time PCR System (Bio-Rad, USA). GAPDH was used as an internal control and the relative 
178 expression levels of mRNA were calculated using the $2^{-\Delta \Delta \mathrm{Ct}}$ method. The primer pairs used in the 179 experiments are listed in Supplementary data 1.

180

181 Prediction of therapeutic agents and target genes.

182 To discover potential anti-fibrotic agents, the identified 185 DEGs were queried using the 183 Connectivity Map online tool (L1000 platform; https://clue.io/11000-query). This tool compares 184 queried signatures with a gene expression profile database of several cell lines after treatment 185 with more than 2000 compounds, most of which are FDA approved. Drugs whose signatures 186 were in opposition to the disease signature were assumed to have therapeutic potential. 


\section{Results}

190 Identification of 185 conserved DEGs.

191

192

193

194

195

196

197

198

199

200

201

202

203

204

205

206

207

208

209

210

211

212

213

214

215

216

217

218

219

220

221

222

223

224

225

226

227

228

As shown in Fig. 1, each dataset was initially analyzed separately to identify DEGs unique to fibrosis of a given origin. 1563 DEGs were identified in GSE6764 (HCV), 243 DEGs in GSE49541 (NAFLD), and 1396 DEGs in GSE84044 (HBV) (Supplementary Data 2). 185 DEGs overlapped across all three datasets, suggesting that these fibrosis-related DEGs may be conserved regardless of disease etiology. Among these 185 DEGs, 174 were up-regulated while only 11 were down-regulated. Interestingly, although the number of DEGs in NAFLD related dataset is relatively small, $94.7 \%$ of the 243 DEGs intersect with other datasets.

\section{Functional enrichment analysis of DEGs.}

In order to compare the differences in gene function among these 3 datasets, GO and KEGG analyses were performed on each dataset (Supplementary Data 3) and top 10 significant GO_BP and KEGG pathways are shown in Table 2 and Table 3, respectively. Unexpectedly, the cell cycle pathway was uniquely enriched in the HBV-related dataset, ranking third among all KEGG pathways for this dataset. When compared with a non-viral fibrosis dataset (GSE49541), those datasets in which fibrosis was of viral origin (GSE6764 and GSE84044) contained DEGs enriched for immune-inflammatory responses, consistent with the distinct role of immunological responses in the initiation and control of local disease in affected individuals.

Next, GO and KEGG analyses were performed on the 185 common DEGs. The GO analysis revealed that most of the proteins encoded by these DEGs were extracellular matrix proteins located in the extracellular space (Fig. 2). The molecular functions (MF) enriched in this dataset were primarily associated with platelet-derived growth-factor binding and extracellular matrix structural constitution, while the enriched biological processes (BP) were primarily those associated with extracellular matrix organization and cell adhesion (Fig. 2). The KEGG analysis revealed that the primary enriched signaling pathways were those associated with ECM-receptor interaction, protein digestion and absorption, focal adhesion, and the PI3K-Akt signaling pathway (Fig. 3). Together, these shared DEGs highlight the central roles of cell-cell adhesion and ECM dysregulation in the development of fibrosis, regardless of the etiological origin of the disease.

\section{PPI network construction and hub gene identification.}

To better understand which of these shared DEGs were most likely to be the key genes most essential for the development of fibrosis, a PPI network for these 185 common DEGs was built with 105 nodes and 275 edges. 80 of the 185 DEGs were not included in the PPI network (Fig. 4), as interaction score of these 80 genes were less than 0.4. Among the 105 genes in the PPI network, the top 25 genes according to the MCC method were selected using the CytoHubba plugin and are sequentially ordered as follows: COL1A2, COL1A1, COL6A3, COL3A1, COL5A2, COL5A1, COL4A1, COL4A2, COL4A3, COL4A4, DCN, COL14A1, LUM, COL15A1, THBS2, FBN1, ITGB8, CDH11, ADAMTS2, CTGF, VCAN, PCOLCE2, SPP1, 
229

230

231

232

233

234

235

236

237

238

239

240

241

242

243

244

245

246

247

248

249

250

251

252

253

254

255

256

257

258

259

260

261

262

263

264

265

266

267

268

VWF, CTSK (Fig. 5). These 25 genes were deemed to be the hub genes and were those genes most likely to be essential for fibrogenesis. Most genes encode ECM components, including COL1A2, DCN, and FBN1. Other hub genes play known roles in ECM structural regulation (THBS2, ITGB8, VWF), while some are associated with ECM degradation (ADAMTS2, PCOLCE2, CTSK). This finding is consistent with known fibrogenic mechanisms, and suggests key potential drug targets that are most likely to have effective anti-fibrotic activity when disrupted.

\section{Hub gene validation.}

In order to extend and validate our findings in a distinct model of human liver fibrosis, these top 25 hub genes were validated in the GSE14323 dataset, in which liver cirrhotic (LC) and normal control tissues (NC) were selected for analysis. Fig. 6 displays a heatmap of GSE14323 expression profile data. This expression profile was consistent with the overlapping DEGs identified in the initial three datasets, with 23 of these 25 hub DEGs being up-regulated in cirrhotic patients. Statistical analysis of these genes in the validation dataset is shown in Fig. 7. Differences for all the hub genes between the LC and NC groups were statistically significant with the exception of ITGB8.

A cell model of liver fibrosis was also constructed to validate these 25 hub genes. When treated with TGF- $\beta 1$, the LX2 cells extended more tentacles and expressed more a-SMA protein (One of the markers of hepatic stellate cell activation) (Fig. 8), indicating the cell model was successfully established. Fig. 9 displays statistical analysis of 25 hub genes relative expression to GAPDH, 13 of these 25 hub genes was up-regulated significantly, which is consistent with the trend of GEO datasets in this study. However, 4 genes (LUM, THBS2, ITGB8 and SPP1) was down-regulated in TGF- $\beta 1$ treated cells. The expression trend of some genes was inconsistent with expectations, likely because TGF- $\beta$-induced hepatic stellate cell activation does not represent the entire activated form or that part of the gene is expressed by other cells such as hepatic parenchymal cells and Kupffer cells.

\section{Prediction of potential therapeutic agents and targets.}

Given the role of 185 DEGs in fibrogenesis, we next wanted to probe for potential therapeutic compounds that might best be suited to target these genes in order to achieve a beneficial therapeutic outcome. To that end the connectivity map L1000 platform, which compiles gene expression profiles associated with a wide range of therapeutic compounds, was used to search for drugs with the potential for therapeutic repurposing as a means of treating liver fibrosis (Supplementary Data 4). The top 10 compounds are shown in Table 4, and when sequentially ordered by median_score are: Prometon, MK-212, Evodiamine, Zosuquidar, CAY-10415, Caffeic-acid, Budesonide, Rilmenidine, Afatinib, Desloratadine. Target genes corresponding to each compound (with the exception of prometon) were also listed. Among these target genes, three (HTR2B, ABCB1 and ALOX5) were significantly up-regulated in HBV and HCV datasets, and the mRNA expression levels in each dataset were listed in Table 5. Together, these 
269 compounds and target genes provide a promising list for researchers or companies interested in 270 conducting pre-clinical research into the mechanisms of and treatments for fibrosis both in vitro 271 and in vivo.

272

273 


\section{Discussion}

275 Globally, HBV, HCV, and NAFLD are the most three common causes of liver fibrosis

276 (Altamirano-Barrera et al. 2017). In the present study, we integrated datasets that were focused

277 on these three most common causes of liver fibrosis, and in so doing we were able to identify

278 different and common signaling pathways for the fibrogenesis.

279 Transition of hepatic stellate cells (HSCs) from a quiescent to an activated state is a sign of 280 the onset of liver fibrosis, and this process is controlled by E-type cyclins (CcnE1, CcnE2) and 281 their associated cyclin-dependent kinase 2 (Cdk2) (Nevzorova et al. 2012; Ohtsubo et al. 1995).

282 Cyclin E-Cdk2 has long been considered an essential master regulator of progression through the 283 G1 phase of the cell cycle (Hwang \& Clurman 2005). According to our KEGG pathway 284 enrichment results, the cell cycle pathway is uniquely enriched in HBV-related fibrosis dataset, 285 with CcnE2 being significantly up-regulated only in this HBV dataset, but not in the HCV or NAFLD datasets (Table 5). Therapeutic targeting of Cyclin E1 via RNAi has been shown to have robust anti-fibrotic activity in mice (Bangen et al. 2017), and if this technology can be applied clinically in future, we predict that it will be most effective in those patients with chronic hepatitis B.

Both HBV and HCV are non-cytopathic viruses, and liver damage in infected individuals is mainly caused by an inflammatory immune response aimed at eliminating the virus (Guidotti \& Chisari 2006), with such persistent inflammation leading to liver fibrosis (Protzer et al. 2012). In contrast to such fibrosis of viral origin, the production of reactive oxygen species (ROS) and resulting oxidative stress is thought to be a critical factor in NAFLD-associated fibrosis. Although NAFLD is always accompanied by an inflammatory reaction with variations in levels of pro-inflammatory cytokines (Cai et al. 2005), the immune-inflammatory response is not significantly enriched in this NAFLD-related dataset, whereas it is clearly enriched in the virusrelated datasets.

By using a PPI network analysis, we identified 25 hub genes, some of which have been previously reported including LUM, THBS2, FBN1(Lou et al. 2017), COL6A3, and COL1A1 (Chen et al. 2017). These 25 hub genes are crucial to fibrogenesis, and are expressed regardless of etiology. The development of anti-fibrotic drugs should therefore focus on these genes as targets. Connective tissue growth factor (CTGF), one of the 25 identified hub genes, is expressed at very low levels in normal liver tissue but is significantly upregulated in fibrotic liver tissue. Recently a clinical trial of patients with HBV-associated liver fibrosis was completed utilizing a human monoclonal antibody reactive to CTGF (NCT01217632). Although this clinical trial was terminated due to an unexpected prominent single-agent effect of entecavir in this patient population, it did show promise.

In this study, we were able to screen for and identify 10 compounds that may have therapeutic activity against liver fibrosis. Among these compounds, evodiamine, caffic-acid, and budesonide have all been shown to be effective in animal models or clinical trials (Alferink et al. 2017; Silveira \& Lindor 2014; Yang et al. 2018; Yang et al. 2017). Four agents, MK-212, CAY10415, afatinib, and desloratadine, have not been tested in vivo, but compounds targeting the 
314 same molecules as these four agents have been reported to have the potential to ameliorate liver 315 fibrosis (Boettcher et al. 2012; Ebrahimkhani et al. 2011; Fuchs et al. 2014; Kennedy et al.

316 2018). Prometon, Zosuquidar, and Rilmeidine have not yet been reported to have relationship

317 with fibrosis.

318 Among the target genes corresponding to these compounds, we found that HTR2B, $319 \mathrm{ABCB} 1$, and ALOX5 were significantly up-regulated in HBV and HCV related liver fibrosis

320

321

322

323

324

325

326

327

328

329

330

331

332

333

334

335

336

337

338

339

340

341

342

343

344

345

346

347

348

349

350

351

352

353 datasets (Data not show). Stimulation of the 5-hydroxytryptamine 2B receptor (HTR2B) on HSCs by serotonin is required to negatively regulate hepatocyte regeneration, and antagonism of HTR2B has been shown to attenuate fibrogenesis and improve liver function in disease models in which fibrosis was pre-established and progressive (Ebrahimkhani et al. 2011). Interestingly, MK-212, an HTR2B agonist, showed a negative liver fibrosis gene expression profile suggesting potential as an anti-fibrotic agent, although formal experimental testing is needed. Arachidonate 5-lipoxygenase (ALOX5) plays a role in the synthesis of leukotrienes from arachidonic acid, and inhibition of the ALOX5 pathway markedly reduces the number of Kupffer cells in culture and attenuates inflammation and fibrosis in experimental liver disease (Titos et al. 2003). Recently, a clinical study revealed that frequent coffee consumption was inversely correlated with liver stiffness (Alferink et al. 2017), with suggestions that the underlying mechanism may be one related to the inhibition of TGF- $\beta 1 / \mathrm{Smad} 3$ signaling and the induction of autophagy in HSCs in response to caffeic acid (Yang et al. 2017). As an inhibitor of ALOX5, caffeic acid may thus be able to attenuate liver fibrosis via this ALOX5 (Sud'ina et al. 1993) pathway.

ATP Binding Cassette Subfamily B Member 1 (ABCB1), is known for encoding P glycoprotein, which is responsible for decreased drug accumulation in multidrug-resistant cells and often mediates the development of resistance to anticancer drugs, such as Zosuquidar mentioned above. However, there are currently no studies reporting that ABCB1, P glycoprotein or Zosuquidar is associated with liver fibrosis. Some studies have reported $\mathrm{P}$ glycoprotein was increased in rat activated HSC (Hannivoort et al. 2008), and its activity was increased by TGF$\beta$ (Baello et al. 2014) and endoplasmic reticulum stress (Ledoux et al. 2003), which are considered to be effective activators of HSC. Combining the findings of our research, we infer that $\mathrm{ABCB} 1$ might be a novel therapeutic target to liver fibrosis, although this hypothesis need to be verified in further study.

\section{Conclusions}

Our study integrated three liver fibrosis datasets, each with fibrosis of a different etiology (HBV, HCV and NAFLD). Through the functional analysis of identified DEGs, we revealed that cell cycle is a pathway uniquely enriched in HBV related dataset and immune-inflammatory response is clearly enriched in the virus-related datasets. We further identified 25 key hub genes, the majority of which were linked to ECM regulation, highlighting the central processes common to all causes of fibrogenesis, offering valuable insights into the conserved nature of fibrotic signaling. Based on the $185 \mathrm{DEGs}$, we were additionally predicted 10 compounds, especially Zosuquidar and corresponding gene target $\mathrm{ABCB} 1$, may have anti-fibrotic activity. While further 
354 experiments will be needed to validate these findings, this successful compound screening effort 355 suggests that it may be possible to repurpose extant drugs to more readily treat liver fibrosis.

356

357

358

359

360 
361

362

363

364

365

366

367

368

369

370

371

372

373

374

375

376

377

378

379

380

381

382

383

384

385

386

387

388

389

390

391

392

393

394

395

396

397

398

399

400

401

402

403

404

405

406

407

408

409

410

\section{References}

Alferink LJM, Fittipaldi J, Kiefte-de Jong JC, Taimr P, Hansen BE, Metselaar HJ, Schoufour JD, Ikram MA, Janssen HLA, Franco OH, and Darwish Murad S. 2017. Coffee and herbal tea consumption is associated with lower liver stiffness in the general population: The Rotterdam study. J Hepatol 67:339-348.

Altamirano-Barrera A, Barranco-Fragoso B, and Mã@Ndez-Sã nN. 2017. Management strategies for liver fibrosis. Annals of Hepatology 16:48-56.

Baello S, lqbal M, Bloise E, Javam M, Gibb W, and Matthews SG. 2014. TGF-beta1 regulation of multidrug resistance P-glycoprotein in the developing male blood-brain barrier. Endocrinology 155:475-484.

Bangen JM, Hammerich L, Sonntag R, Baues M, Haas U, Lambertz D, Longerich T, Lammers T, Tacke F, Trautwein C, and Liedtke C. 2017. Targeting CCl4 -induced liver fibrosis by RNA interference-mediated inhibition of cyclin E1 in mice. Hepatology 66:1242-1257.

Boettcher E, Csako G, Pucino F, Wesley R, and Loomba R. 2012. Meta-analysis: pioglitazone improves liver histology and fibrosis in patients with non-alcoholic steatohepatitis. Aliment Pharmacol Ther 35:66-75.

Bottcher K, and Pinzani M. 2017. Pathophysiology of liver fibrosis and the methodological barriers to the development of anti-fibrogenic agents. Adv Drug Deliv Rev 121:3-8.

Cai D, Yuan M, Frantz DF, Melendez PA, Hansen L, Lee J, and Shoelson SE. 2005. Local and systemic insulin resistance resulting from hepatic activation of IKK-beta and NF-kappaB. Nat Med 11:183-190.

Chan KM, Wu TH, Wu TJ, Chou HS, Yu MC, and Lee WC. 2016. Bioinformatics microarray analysis and identification of gene expression profiles associated with cirrhotic liver. Kaohsiung J Med Sci 32:165-176.

Chen W, Zhao W, Yang A, Xu A, Wang H, Cong M, Liu T, Wang P, and You H. 2017. Integrated analysis of microRNA and gene expression profiles reveals a functional regulatory module associated with liver fibrosis. Gene 636:87-95.

Djordjevic DB, Zdravkovic M, Nagorni A, Manolis A, Tsioufis C, and Lovic D. 2018. A Critical Approach of Guideline Therapeutic Recommendations for NAFLD. Current vascular pharmacology 16:228-238.

Ebrahimkhani MR, Oakley F, Murphy LB, Mann J, Moles A, Perugorria MJ, Ellis E, Lakey AF, Burt AD, Douglass A, Wright MC, White SA, Jaffre F, Maroteaux L, and Mann DA. 2011. Stimulating healthy tissue regeneration by targeting the $5-\mathrm{HT}(2) \mathrm{B}$ receptor in chronic liver disease. Nat Med 17:1668-1673.

Feng R, Yuan X, Shao C, Ding H, Liebe R, and Weng HL. 2018. Are we any closer to treating liver fibrosis (and if no, why not)? Journal of digestive diseases 19:118-126.

Fuchs BC, Hoshida Y, Fujii T, Wei L, Yamada S, Lauwers GY, McGinn CM, DePeralta DK, Chen X, Kuroda T, Lanuti M, Schmitt AD, Gupta S, Crenshaw A, Onofrio R, Taylor B, Winckler W, Bardeesy N, Caravan P, Golub TR, and Tanabe KK. 2014. Epidermal growth factor receptor inhibition attenuates liver fibrosis and development of hepatocellular carcinoma. Hepatology 59:1577-1590.

Guidotti LG, and Chisari FV. 2006. Immunobiology and pathogenesis of viral hepatitis. Annu Rev Pathol 1:23-61.

Hannivoort RA, Dunning S, Vander Borght S, Schroyen B, Woudenberg J, Oakley F, BuistHoman M, van den Heuvel FA, Geuken M, Geerts A, Roskams T, Faber KN, and Moshage $H$. 2008. Multidrug resistance-associated proteins are crucial for the viability of activated rat hepatic stellate cells. Hepatology 48:624-634.

Hwang HC, and Clurman BE. 2005. Cyclin E in normal and neoplastic cell cycles. Oncogene 24:2776-2786.

Ji D, Chen GF, Wang JC, Cao LH, Lu F, Mu XX, Zhang XY, and Lu XJ. 2018. Identification of 
411

412

413

414

415

416

417

418

419

420

421

422

423

424

425

426

427

428

429

430

431

432

433

434

435

436

437

438

439

440

441

442

443

444

445

446

447

448

449

450

451

452

453

454

455

456

457

458

459

460

461

TAF1, HNF4A, and CALM2 as potential therapeutic target genes for liver fibrosis. J Cell Physiol.

Kennedy L, Hargrove L, Demieville J, Karstens W, Jones H, DeMorrow S, Meng F, Invernizzi P, Bernuzzi F, Alpini G, Smith S, Akers A, Meadows V, and Francis H. 2018. Blocking $\mathrm{H} 1 / \mathrm{H} 2$ histamine receptors inhibits damage/fibrosis in Mdr2(-/-) mice and human cholangiocarcinoma tumorigenesis. Hepatology.

Ledoux S, Yang R, Friedlander G, and Laouari D. 2003. Glucose depletion enhances Pglycoprotein expression in hepatoma cells: role of endoplasmic reticulum stress response. Cancer Res 63:7284-7290.

Liver EAFTSOT. 2012. EASL clinical practical guidelines: management of alcoholic liver disease. Journal of hepatology 57:399-420.

Liver EAFTSOT. 2017a. EASL 2017 Clinical Practice Guidelines on the management of hepatitis B virus infection. Journal of hepatology 67:370-398.

Liver EAfTSoT. 2017b. EASL recommendations on treatment of hepatitis C 2016. Journal of hepatology 66:153.

Lou Y, Tian GY, Song Y, Liu YL, Chen YD, Shi JP, and Yang J. 2017. Characterization of transcriptional modules related to fibrosing-NAFLD progression. Sci Rep 7:4748.

Mas VR, Maluf DG, Archer KJ, Yanek K, Kong X, Kulik L, Freise CE, Olthoff KM, Ghobrial RM, Mclver P, and Fisher R. 2009. Genes involved in viral carcinogenesis and tumor initiation in hepatitis $C$ virus-induced hepatocellular carcinoma. Mol Med 15:85-94.

Moylan CA, Pang H, Dellinger A, Suzuki A, Garrett ME, Guy CD, Murphy SK, Ashley-Koch AE, Choi SS, Michelotti GA, Hampton DD, Chen Y, Tillmann HL, Hauser MA, Abdelmalek MF, and Diehl AM. 2014. Hepatic gene expression profiles differentiate presymptomatic patients with mild versus severe nonalcoholic fatty liver disease. Hepatology 59:471-482.

Nevzorova YA, Bangen JM, Hu W, Haas U, Weiskirchen R, Gassler N, Huss S, Tacke F, Sicinski P, Trautwein C, and Liedtke C. 2012. Cyclin E1 controls proliferation of hepatic stellate cells and is essential for liver fibrogenesis in mice. Hepatology 56:1140-1149.

Ohtsubo M, Theodoras AM, Schumacher J, Roberts JM, and Pagano M. 1995. Human cyclin E, a nuclear protein essential for the G1-to-S phase transition. Mol Cell Biol 15:2612-2624.

Protzer U, Maini MK, and Knolle PA. 2012. Living in the liver: hepatic infections. Nat Rev Immunol 12:201-213.

Silveira MG, and Lindor KD. 2014. Obeticholic acid and budesonide for the treatment of primary biliary cirrhosis. Expert Opinion on Pharmacotherapy 15:365-372.

Sud'ina GF, Mirzoeva OK, Pushkareva MA, Korshunova GA, Sumbatyan NV, and Varfolomeev SD. 1993. Caffeic acid phenethyl ester as a lipoxygenase inhibitor with antioxidant properties. FEBS Lett 329:21-24.

Titos E, Claria J, Planaguma A, Lopez-Parra M, Villamor N, Parrizas M, Carrio A, Miquel R, Jimenez W, Arroyo V, Rivera F, and Rodes J. 2003. Inhibition of 5-lipoxygenase induces cell growth arrest and apoptosis in rat Kupffer cells: implications for liver fibrosis. FASEB J 17:1745-1747.

Tsochatzis EA, Bosch J, and Burroughs AK. 2014. Liver cirrhosis. The Lancet 383:1749-1761.

Wang M, Gong Q, Zhang J, Chen L, Zhang Z, Lu L, Yu D, Han Y, Zhang D, Chen P, Zhang X, Yuan Z, Huang J, and Zhang X. 2017. Characterization of gene expression profiles in HBV-related liver fibrosis patients and identification of ITGBL1 as a key regulator of fibrogenesis. Sci Rep 7:43446.

Wurmbach E, Chen YB, Khitrov G, Zhang W, Roayaie S, Schwartz M, Fiel I, Thung S, Mazzaferro V, Bruix J, Bottinger E, Friedman S, Waxman S, and Llovet JM. 2007. Genome-wide molecular profiles of HCV-induced dysplasia and hepatocellular carcinoma. Hepatology 45:938-947.

Yang D, Li L, Qian S, and Liu L. 2018. Evodiamine ameliorates liver fibrosis in rats via TGFbeta1/Smad signaling pathway. J Nat Med 72:145-154. 
462 Yang N, Dang S, Shi J, Wu F, Li M, Zhang X, Li Y, Jia X, and Zhai S. 2017. Caffeic acid 463 phenethyl ester attenuates liver fibrosis via inhibition of TGF-beta1/Smad3 pathway and induction of autophagy pathway. Biochem Biophys Res Commun 486:22-28.

465 


\section{Figure 1}

Venn diagram of DEGs.

Venn diagram of DEGs from the 3 cohort profile sets (GSE6764, GSE49541, GSE84044), generated using an online tool. Each colored circle represents a different dataset, and areas of overlap indicate shared DEGs. Statistically significant DEGs were defined based on adj.p < 0.05 and $[\mathrm{FC}]>1.5$ as the cut-off criteria. 


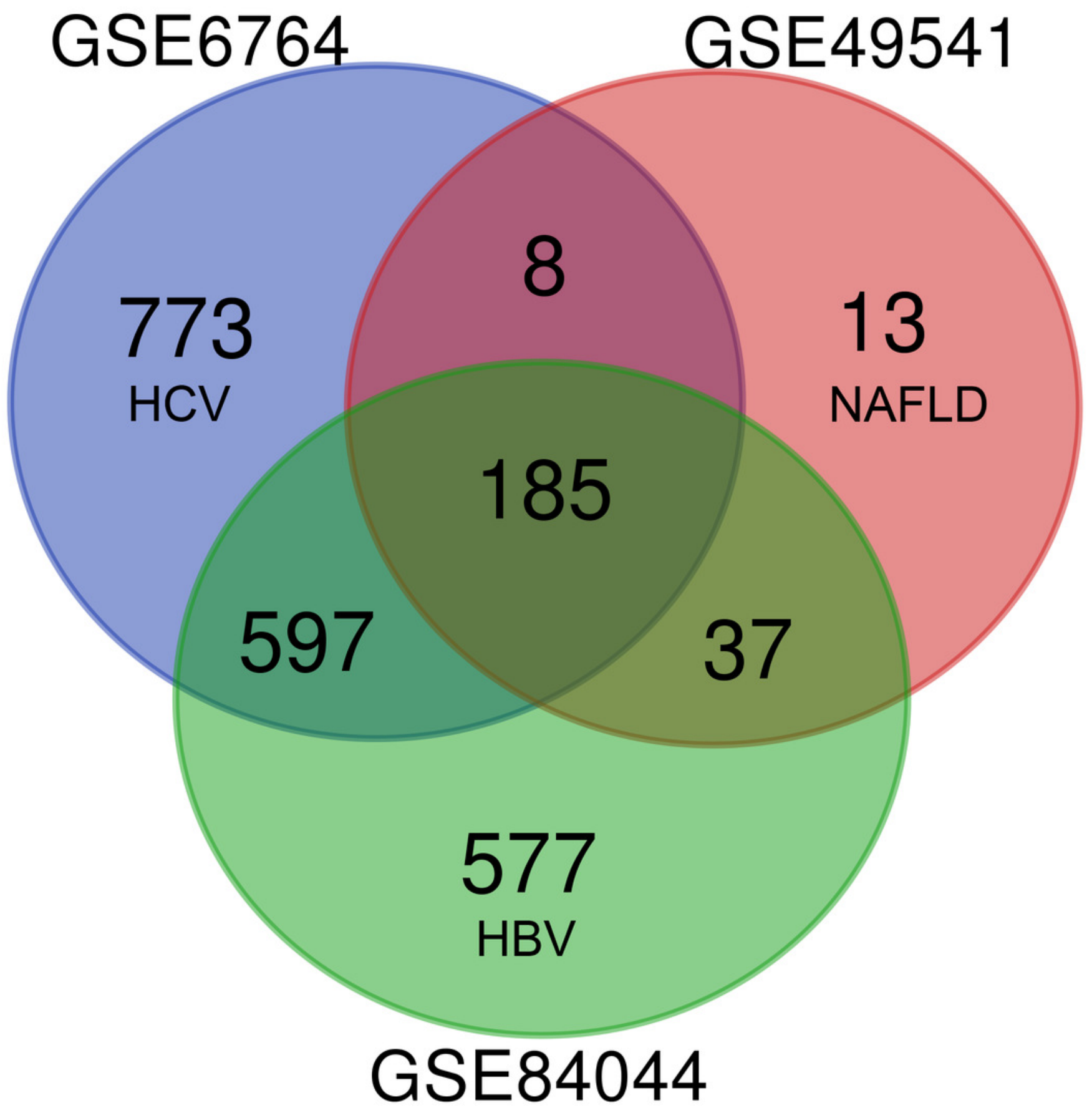


Figure 2 (on next page)

GO enrichment analyses of 158 common DEGs.

The Top 10 terms in each GO category (MF: Molecular function, CC: Cellular components, BP: Biological processes). 
Figure 3 (on next page)

KEGG enrichment analyses of 158 common DEGs.

All significant KEGG pathways. GO and KEGG analysis was performed using the DAVID online tool with the cutoff criteria of FDR $<0.05$. The color of each bubble represents the FDR for that term, with red representing greater significance. The rich factor refers to the proportion of enriched genes for each term. 
Figure 4 (on next page)

Protein-protein interaction (PPI) network complex.

Using the STRING online database, a total of 105 DEGs (101 up-regulated genes shown in Red and 4 down-regulated genes shown in Green) were filtered into a DEG PPI network complex. The gray line between two proteins means an interaction score no less than 0.4 , and the more interactions with other proteins, the more important this protein is. 
Peer

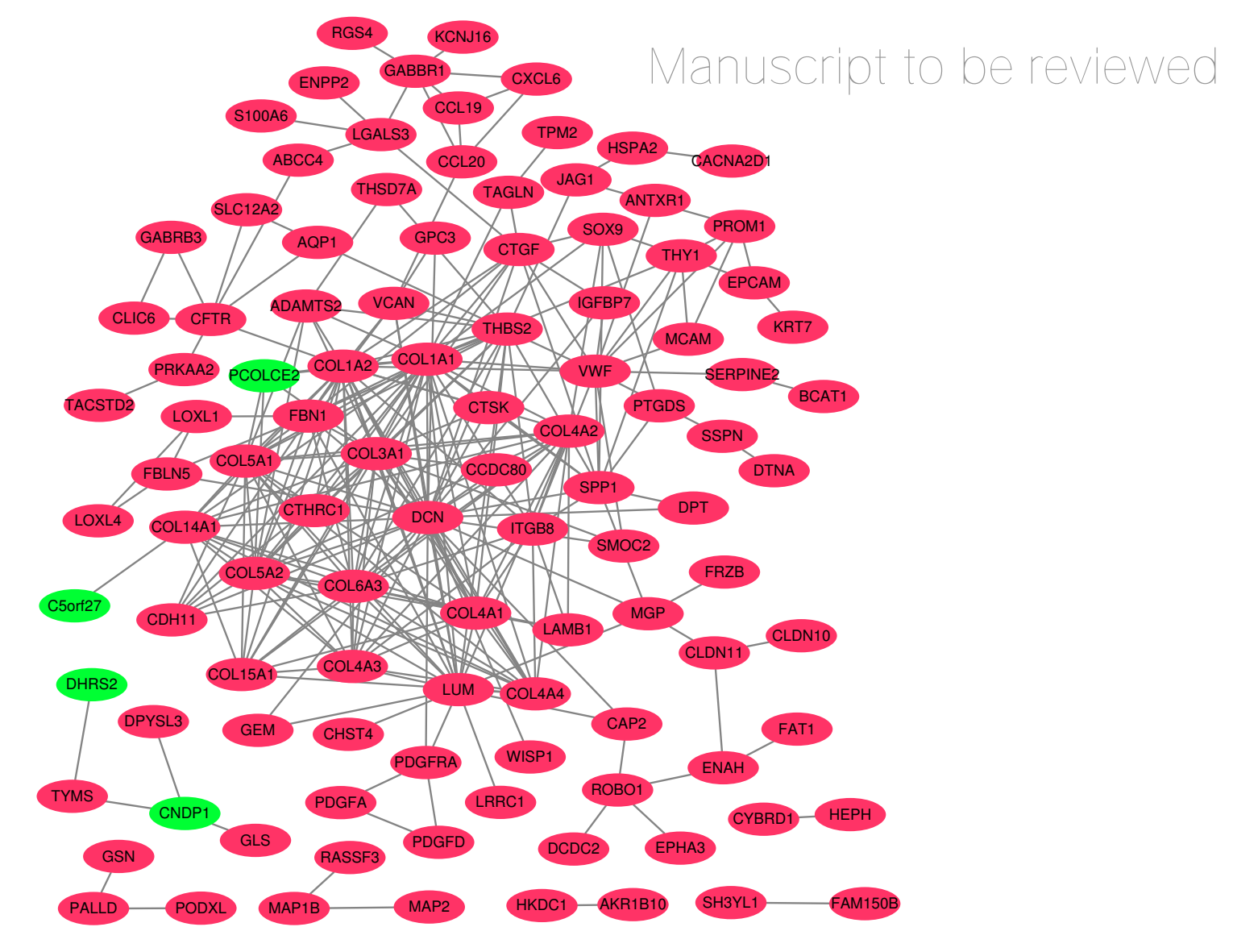

Peer) reviewing PDF | (2018:12:33585:1:0:NEW 5 Feb 2019) 
Figure $\mathbf{5}$ (on next page)

Top 25 hub gene network.

The top 25 genes derived from the MMC method were chosen using the CytoHubba plugin. Advanced ranking is represented by a redder color. 


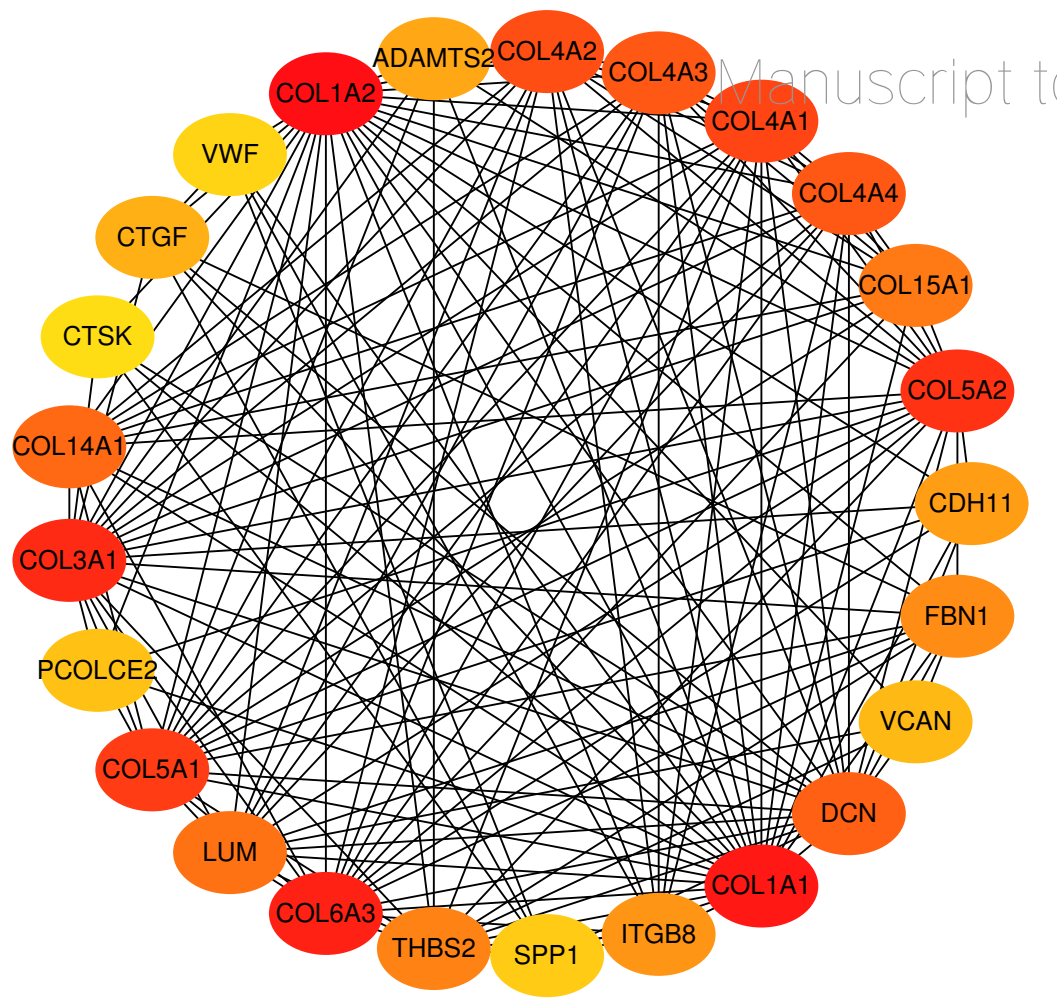


Figure 6

Heatmap of the expression of the 25 hub genes in the GSE14323 validation dataset.

Red colored: up-regulated; Green colored: down-regulated.

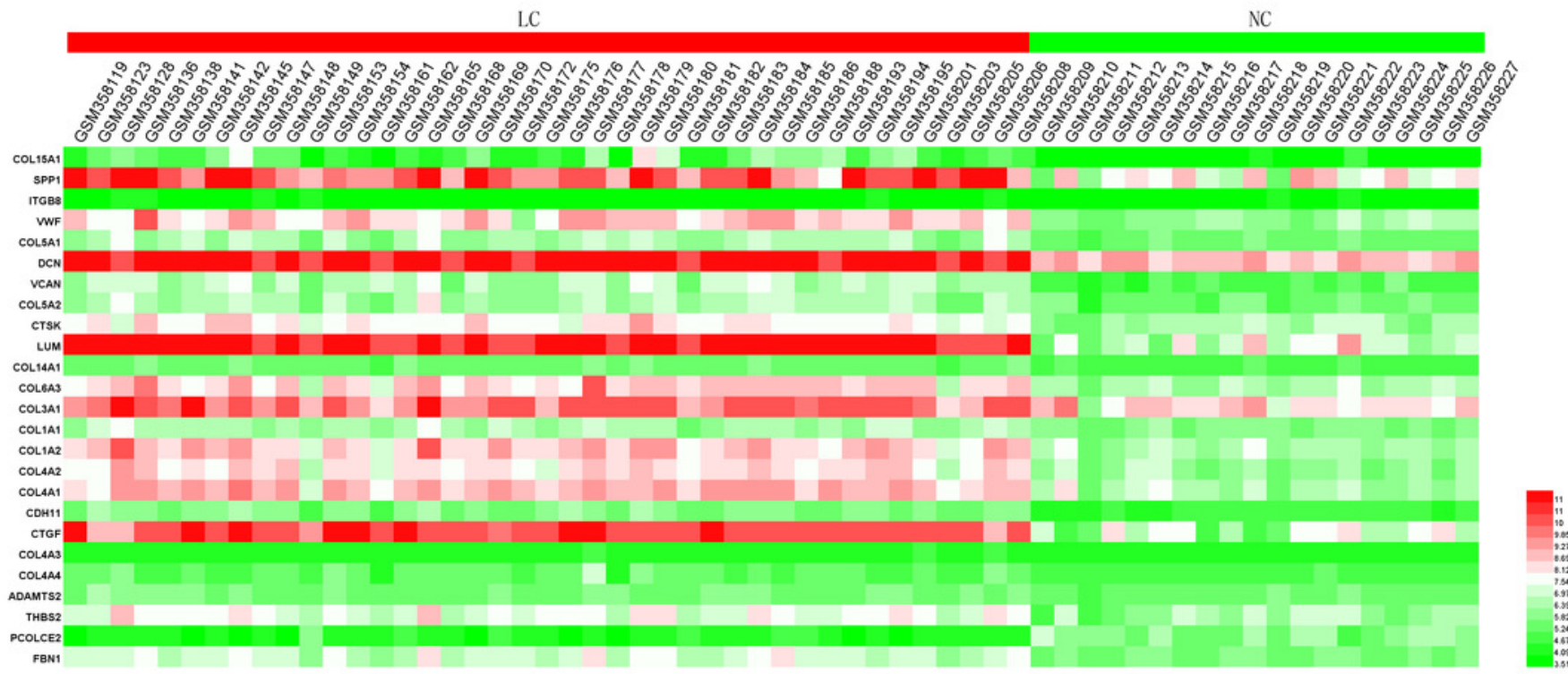




\section{Figure 7 (on next page)}

Statistical analysis of the expression of 25 hub genes in GSE14323.

The differences in expression of all hub genes between the liver cirrhosis (LC) group and the normal control (NC) group were statistically significant with the exception of ITGB8. LC: liver cirrhosis; NC: normal control. ${ }^{*} p<0.05,{ }^{* *} p<0.01,{ }^{* * *} p<0.001$. 
Figure 8

Successful construction of liver fibrosis cell model.

After treated with TGF- $\beta 1$ for 24 hours $\square$ the LX2 cells morphology became irregular and extended more ten(A)tacles, and expressed more a-SMA protein (One of the markers of hepatic stellate cell activation) determined by Western Blot (B).

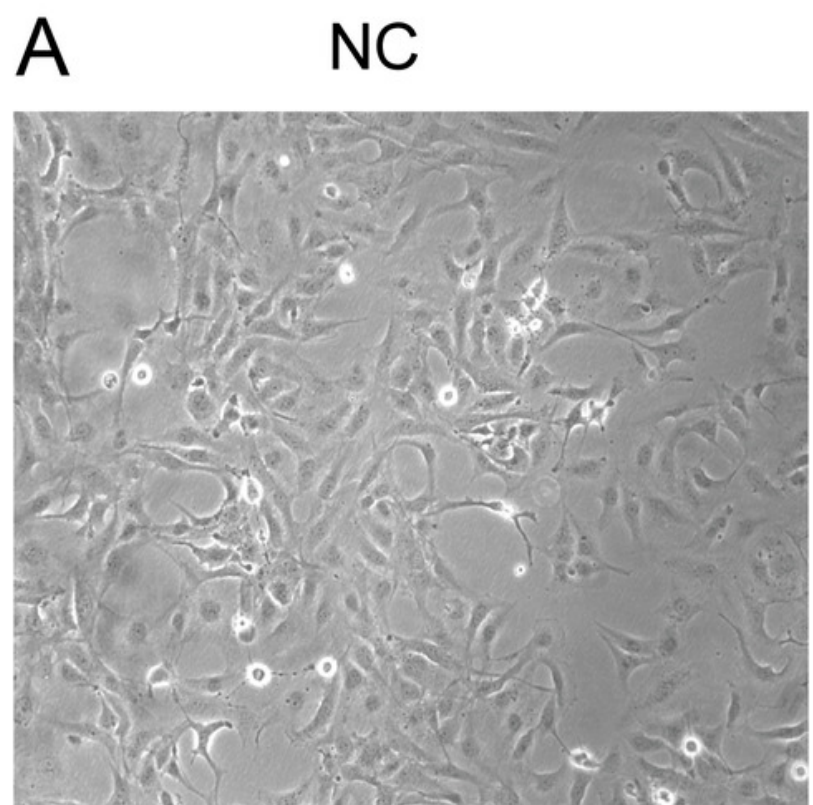

TGF- $\beta$

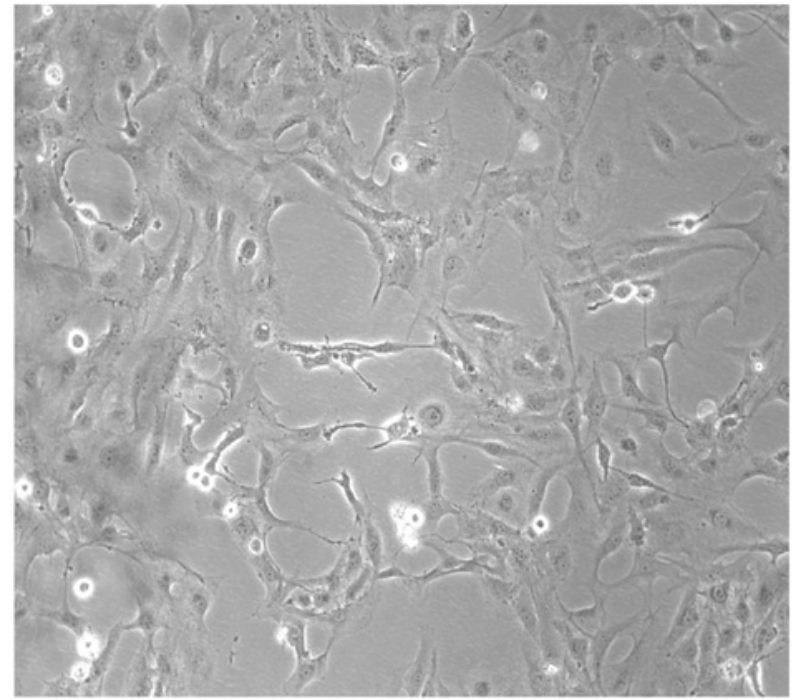

B

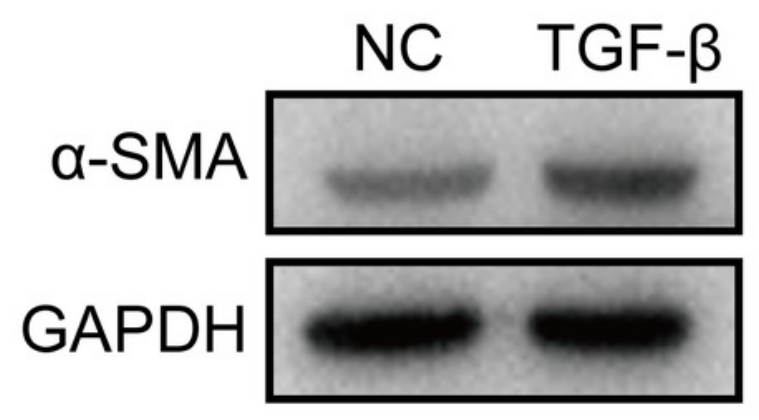




\section{Figure 9 (on next page)}

mRNA expressions of 25 hub genes in cell model by qPCR.

When LX2 cells were activated by TGF- $\beta 1 \square 13$ genes significantly up-regulated which is consistent with the trend of microarray data in this study. 4 genes (LUM, THBS2, ITGB8 and SPP1) was down-regulated. 


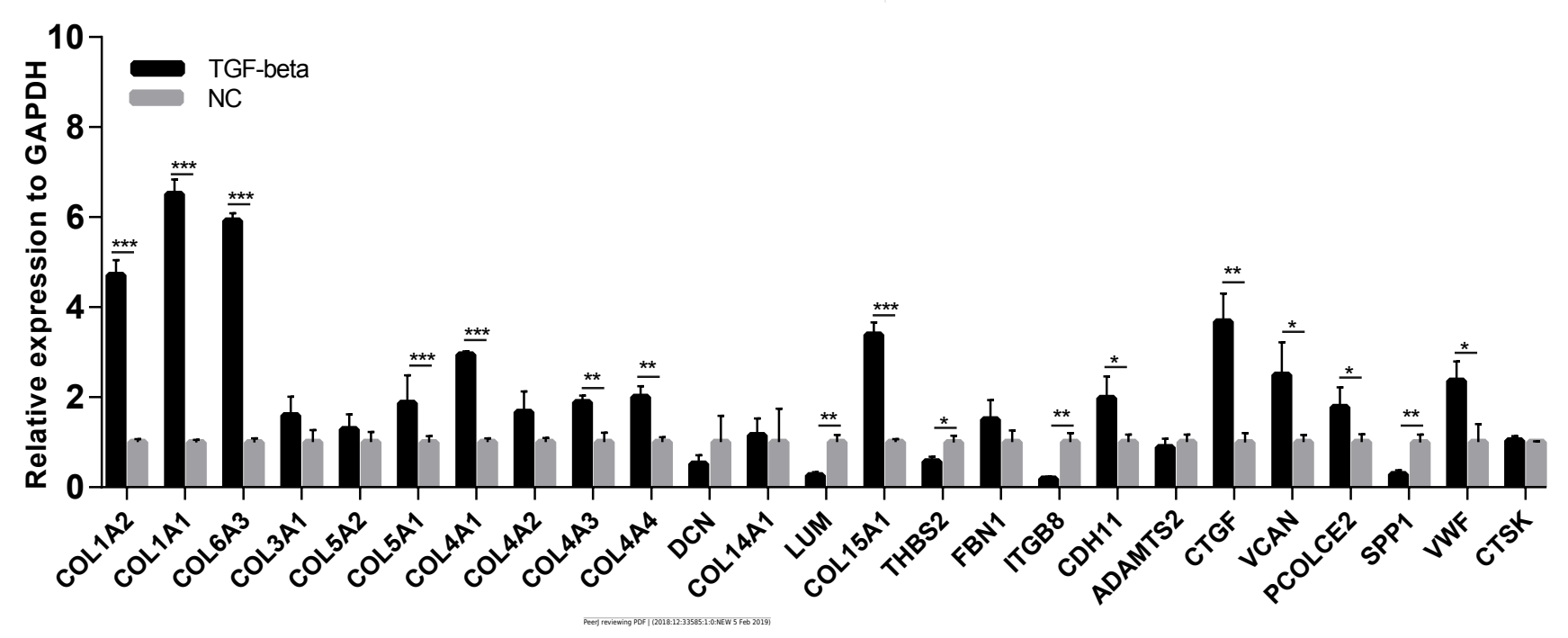




\section{Table $\mathbf{1}$ (on next page)}

Accession information for datasets downloaded from the GEO database.

GSE6764, GSE49541 and GSE84044 was used for identifying DEGs; GSE14323 was used for validation. 


\begin{tabular}{lcccccc}
\hline Accession & GPL & Etiology & $\begin{array}{c}\text { Sample Size } \\
\text { Case/control }\end{array}$ & $\begin{array}{c}\text { Sample Fibrosis } \\
\text { Stage }\end{array}$ & Country & Year \\
\hline GSE6764 & GPL570 & HCV & $10 / 13$ & F4/FO & USA & 2007 \\
GSE49541 & GPL570 & NAFLD & $32 / 40$ & F3-F4/F0-F1 & USA & 2013 \\
GSE84044 & GPL570 & HBV & $28 / 63$ & F3-F4/F0-F1 & China & 2016 \\
GSE14323 & GPL571 & HCV & $41 / 9$ & F4/F0 & USA & 2009 \\
\hline
\end{tabular}

1 


\section{Table 2 (on next page)}

Top 10 GO_BP terms in each datasets ordered by FDR.

Count: number of genes enriched in the corresponding pathway; FDR, false discovery rate. 


\begin{tabular}{|c|c|c|c|}
\hline GO ID & Biological process & Count & FDR \\
\hline \multicolumn{4}{|c|}{ GSE6764-HCV } \\
\hline GO:0030198 & extracellular matrix organization & $59 / 196$ & $8.35 \mathrm{E}-17$ \\
\hline GO:0060337 & type I interferon signaling pathway & $33 / 64$ & $3.82 \mathrm{E}-16$ \\
\hline GO:0006955 & immune response & $88 / 421$ & $7.84 \mathrm{E}-15$ \\
\hline GO:0051607 & defense response to virus & $47 / 165$ & $9.18 \mathrm{E}-12$ \\
\hline GO:0060333 & interferon-gamma-mediated signaling pathway & $30 / 71$ & $1.73 \mathrm{E}-11$ \\
\hline GO:0007155 & cell adhesion & $79 / 459$ & $2.87 \mathrm{E}-08$ \\
\hline GO:0007165 & signal transduction & $144 / 1161$ & $9.27 \mathrm{E}-06$ \\
\hline GO:0050900 & leukocyte migration & $31 / 122$ & $1.34 \mathrm{E}-05$ \\
\hline GO:0045071 & negative regulation of viral genome replication & $17 / 40$ & $2.56 \mathrm{E}-05$ \\
\hline GO:0042493 & response to drug & $53 / 304$ & $5.86 \mathrm{E}-05$ \\
\hline \multicolumn{4}{|c|}{ GSE49541-NAFLD } \\
\hline GO:0030198 & extracellular matrix organization & $29 / 196$ & $6.55 \mathrm{E}-18$ \\
\hline GO:0007155 & cell adhesion & $37 / 459$ & $7.26 \mathrm{E}-15$ \\
\hline GO:0030574 & collagen catabolic process & $13 / 64$ & $6.27 \mathrm{E}-08$ \\
\hline GO:0030199 & collagen fibril organization & $9 / 39$ & $5.87 \mathrm{E}-05$ \\
\hline \multicolumn{4}{|c|}{ GSE84044-HBV } \\
\hline GO:0006955 & immune response & $87 / 421$ & $2.1693 \mathrm{E}-16$ \\
\hline GO:0007155 & cell adhesion & $81 / 459$ & $7.1809 \mathrm{E}-11$ \\
\hline GO:0030198 & extracellular matrix organization & $46 / 196$ & $2.2322 \mathrm{E}-09$ \\
\hline GO:0070374 & positive regulation of ERK1 and ERK2 cascade & $38 / 175$ & $2.6745 \mathrm{E}-06$ \\
\hline GO:0070098 & chemokine-mediated signaling pathway & $23 / 71$ & $3.4269 \mathrm{E}-06$ \\
\hline GO:0002250 & adaptive immune response & $33 / 148$ & $1.927 \mathrm{E}-05$ \\
\hline GO:0060326 & cell chemotaxis & $21 / 65$ & $2.1359 \mathrm{E}-05$ \\
\hline GO:0006954 & inflammatory response & $58 / 379$ & $9.3052 \mathrm{E}-05$ \\
\hline GO:0002548 & monocyte chemotaxis & $16 / 42$ & 0.00015641 \\
\hline GO:0030574 & collagen catabolic process & $19 / 64$ & 0.00052759 \\
\hline
\end{tabular}

1 


\section{Table 3(on next page)}

Top 10 KEGG pathways in each dataset ordered by FDR.

*Pathway is unique in the corresponding dataset. 


\begin{tabular}{|c|c|c|c|}
\hline KEGG ID & Pathway & Count & FDR \\
\hline \multicolumn{4}{|c|}{ GSE6764-HCV } \\
\hline hsa04514 & Cell adhesion molecules (CAMs) & $44 / 142$ & $2.43 \mathrm{E}-10$ \\
\hline hsa05332 & Graft-versus-host disease & $18 / 33$ & 4.53E-07 \\
\hline hsa05330 & Allograft rejection & $18 / 37$ & $4.39 \mathrm{E}-06$ \\
\hline hsa04940 & Type I diabetes mellitus & $19 / 42$ & $6.01 \mathrm{E}-06$ \\
\hline hsa05416 & Viral myocarditis & $22 / 57$ & 8.34E-06 \\
\hline hsa04510 & Focal adhesion & $46 / 206$ & $1.08 \mathrm{E}-05$ \\
\hline hsa04512 & ECM-receptor interaction & $27 / 87$ & $2.20 \mathrm{E}-05$ \\
\hline hsa04612 & Antigen processing and presentation & $25 / 76$ & $2.37 \mathrm{E}-05$ \\
\hline hsa05164 & Influenza A & $39 / 174$ & $1.64 \mathrm{E}-04$ \\
\hline hsa05168 & Herpes simplex infection & $40 / 183$ & $2.22 \mathrm{E}-04$ \\
\hline \multicolumn{4}{|c|}{ GSE49541-NAFLD } \\
\hline hsa04512 & ECM-receptor interaction & $17 / 87$ & $3.17 \mathrm{E}-10$ \\
\hline hsa04510 & Focal adhesion & $21 / 206$ & $6.22 \mathrm{E}-08$ \\
\hline hsa04974 & Protein digestion and absorption & $13 / 88$ & $1.32 \mathrm{E}-05$ \\
\hline hsa04151 & PI3K-Akt signaling pathway & $22 / 345$ & $9.83 \mathrm{E}-05$ \\
\hline hsa05146 & Amoebiasis & $11 / 106$ & 0.007509 \\
\hline \multicolumn{4}{|c|}{ GSE84044-HBV } \\
\hline hsa04512 & ECM-receptor interaction & $28 / 87$ & 7.34E-07 \\
\hline hsa05323 & Rheumatoid arthritis & $27 / 88$ & $5.01 \mathrm{E}-06$ \\
\hline hsa04110 & Cell cycle* & $32 / 124$ & $1.4 \mathrm{E}-05$ \\
\hline hsa04672 & Intestinal immune network for IgA production & $18 / 47$ & $9.61 \mathrm{E}-05$ \\
\hline hsa05150 & Staphylococcus aureus infection & $19 / 54$ & 0.000169 \\
\hline hsa05222 & Small cell lung cancer & $23 / 85$ & 0.001041 \\
\hline hsa04151 & PI3K-Akt signaling pathway & $56 / 345$ & 0.001159 \\
\hline hsa04510 & Focal adhesion & $39 / 206$ & 0.00179 \\
\hline hsa05166 & HTLV-I infection & $45 / 256$ & 0.00197 \\
\hline hsa04974 & Protein digestion and absorption & $23 / 88$ & 0.001973 \\
\hline
\end{tabular}

1 
Table 4 (on next page)

Top 10 compounds predicted to have activity against liver fibrosis as predicted via connectivity map.

*Targets were matched to DEGs in selected datasets. 


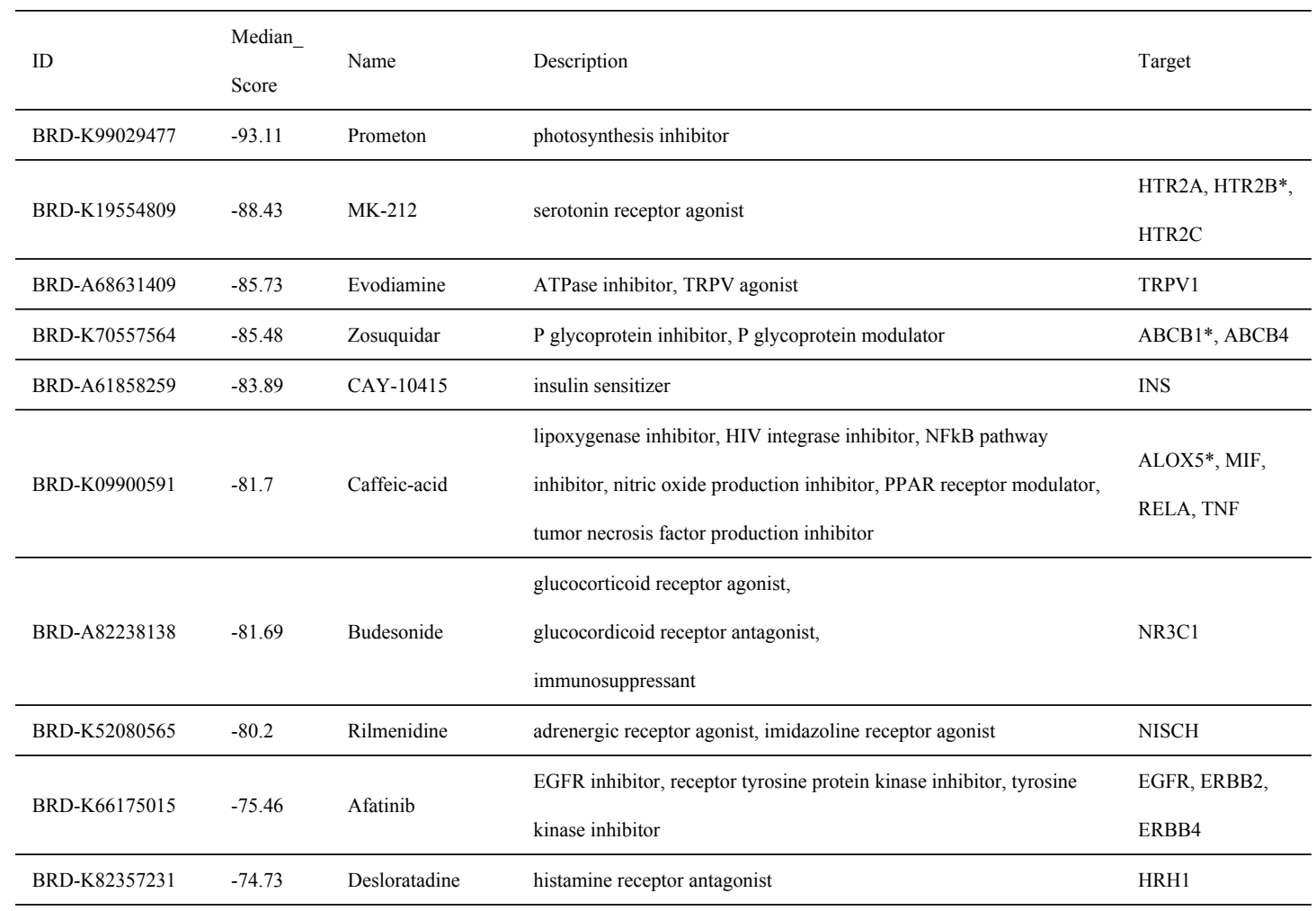

1 


\section{Table 5 (on next page)}

mRNA expression levels of selected genes in each datasets

logFC: $\log 2$ (Fold Change); Genes with an expression level of $|\log F C|>0.585$ and adj.P.Val $<$ 0.05 were deemed to be the DEGs in this study. 


\begin{tabular}{|c|c|c|c|c|c|c|}
\hline \multirow{2}{*}{$\begin{array}{c}\text { Gene } \\
\text { symbol }\end{array}$} & \multicolumn{2}{|c|}{ GSE6764 (HCV) } & \multicolumn{2}{|c|}{ GSE49541 (NAFLD) } & \multicolumn{2}{|c|}{ GSE84044 (HBV) } \\
\hline & $\log F C$ & adj.P.Val & $\log \mathrm{FC}$ & adj.P.Val & $\log F C$ & adj.P.Val \\
\hline HTR2B & 1.24 & $1.63 \mathrm{E}-02$ & 0.33 & $2.26 \mathrm{E}-01$ & 0.74 & $2.61 \mathrm{E}-06$ \\
\hline ABCB1 & 0.75 & $2.24 \mathrm{E}-02$ & 0.26 & $1.75 \mathrm{E}-01$ & 0.76 & $9.61 \mathrm{E}-08$ \\
\hline ALOX5 & 0.99 & $2.88 \mathrm{E}-02$ & 0.23 & $2.72 \mathrm{E}-01$ & 1.06 & 7.79E-09 \\
\hline CcnE2 & -0.39 & $1.09 \mathrm{E}-01$ & 0.46 & $4.36 \mathrm{E}-02$ & 0.96 & $9.91 \mathrm{E}-07$ \\
\hline
\end{tabular}

1 